\title{
Survey on knowledge based methods to assist fault restoration in power distribution networks
}

\author{
Y. Oualmakran ${ }^{1}$, J. Meléndez ${ }^{1}$, S.Herraiz ${ }^{1}$, Mercedes López-Perea ${ }^{2}$ and Eloy González ${ }^{2}$ \\ ${ }^{1}$ Institut d'Informàtica i Aplicacions \\ Research group eXiT \\ Universitat de Girona \\ Campus of Montilivi - Av. Lluis Santaló s/n, 17003 Girona (Spain) \\ Phone/Fax number: +0034 972418883 /+0034 972418259
}

e-mail: youssef.oualmakran@udg.edu, joaquim.melendez@silver.udg.edu , sergio.herraiz@silver.udg.edu

\author{
${ }^{2}$ Indra Software Labs \\ Madrid \\ C/Acanto 11, 28045 Madrid (Spain) \\ Phone: +34912578944 \\ e-mail: mlopezperea@indra.es, egonzalezort@indra.es
}

\begin{abstract}
The motivation behind this paper is the many changes that happen in power distribution networks, theoretical developments, and their consequences on power distribution restoration. The stages of this process are defined with a focus on steps taken after the fault isolation. The final purpose of the operators is defined, as are the rules which must be strictly respected. Power quality issues are stressed. Moreover, recent techniques (metaheuristic and learning methods) are discussed. Heuristic rules also known as expert rules or knowledge based rules used in power restoration are outlined for their supportive role. Finally, a comparison of some selected papers is given. This enables to illustrate how the defined goals and changes in the network are reflected in recent literature. Our conclusion from the study of the state of the art is that there is still a lot of work to be done in developing novel techniques, integrating the goals and inserting the evolving features of power distribution networks.
\end{abstract}

\section{Key words}

Distribution System Restoration, Artificial intelligence, Power Quality, Reliability index, Metaheuristic

\section{Introduction}

This paper analyses knowledge based strategies proposed in the literature for power restoration in distribution systems and gives a comparison of different goals and restrictions dealt in the different papers. We consider the stricter meaning of restoration, as many papers do: the reduction of the power outage zone after the fault has been isolated.
The last significant review on power restoration was presented ten years ago and presented the maturity of the field [1]. There is also an older paper reviewing restoration techniques [2]. But recently distribution systems have experienced a radical change due to the progress of distributed generation which can impact the reliability indexes [3], [4]. This change will be bigger when SmartGrids are definitely implanted (electric vehicles, energy storage, smart metering and smart field devices, active demand management, etc.). At the same time, data management and related artificial intelligence techniques have also evolved offering a variety of methods for data mining and knowledge discovery that can be introduced in the decision support systems.

Restoration goals, strategies, constraints and methods are analyzed from a reliability and power quality perspective. Sudden disturbances caused by weather and environment (e.g. tree flashover), unbalance between demand and generation, plant failures, shortage of plant capacity and other similar incidents can result in power quality problems or a loss of supply in the power distribution systems [5]. Basic stages to recover power involve fault detection, diagnostic, isolation, restoration, reconfiguration and reparation. But their execution can obey to different goals and strategies and have implication on quality of delivered power. General principles to be considered in the restoration procedures and common used guidelines are put together with knowledge based solutions and methods recently proposed to support operators decisions. This paper is organized in three additional sections. The following 
section reviews the basic stages, goals and constraints. Next, knowledge based techniques are explained. Then, a comparison o selected papers is given. Finally, we conclude with the contributions of this paper and future possible work.

\section{Power Restoration: stages, goals and constraints}

The restoration process happens when there is an outage or loss of power. To restore power, the operator proceeds to several steps called stages in the best way (i.e. that optimizes the goals) while respecting operating and engineering limitations (i.e. the constraints). In this section, we will first describe briefly the main stages, then explain the main goals and finally outline the restrictions.

\section{A. Stages}

When a fault happens in the network, the SCADA informs the operator who proceeds sequentially to the location, isolation and restoration stages. However, usually rural areas and low voltage networks are neither fully automated nor entirely monitored. Fault occurrences in this case are notified by affected clients. The location is deduced from clusters of calls in combination with crew inspection coordinated with the control center. Curcic et al. give a more detailed decomposition: diagnostic, location, isolation, searching for the restoration solution, implementing the restoration solution and post-restoration stage [2]. This last stage deals with less urgent goals such as load balancing, improving power quality and minimizing operational costs.

The power distribution system operates normally in radial topology, but the tie-switches give them a physical meshed structure. Restoration is done through network reconfiguration by operating circuit breaker and tie switches. In the process alternate routes for the power flow are created. This can also include capacitor switches [6]. Finding the best reconfiguration network requires complying with the constraints while optimizing the goals.

This may entail conflicts. Thus trade-offs have to be made. The stages are largely sequential but not in a strict manner. Diagnostic may be optional or happen at the end. The basic stages are:

- Detection: the EMS, SCADA or customers notify the distribution company that there is a fault.

- Diagnostic: the operator in collaboration with the crew looks for the cause of the fault.

- Location: they try to find where it has happened.

- Isolation: the fault is isolated as narrowly as possible by opening tie-switches.

- Restoration: the aim is to restore as much power as possible and as quickly as possible.
- $\quad$ Post-restoration or reconfiguration: the operating costs are reduced and power quality is improved. There is no standard name. This stage is sometimes mixed with restoration.

- Reparation: the cause is fixed. Reparation is not needed when the fault is transient.

\section{B. Goals}

The utility has to make a trade-off between targets that increase customer satisfaction and those that reduce operational cost. This dilemma existed for several decades and it is not expected to disappear.

- Outage size: it can be expressed in several ways (number of customers, amount of energy). Priority customers should receive special attention. Hongshun and Xiangfei proposed a system with four types of customers according to the category of consequences that a power outage can entail: human life in danger (e.g. hospitals), serious financial damage or affection to a large community (e.g. banks), inconvenience or public concern (e.g. schools) [7]. In a fourth category are put the normal customers.

- Restoration speed: the restoration should happen as quickly as possible (the solution and the implementation should be fast). Planned and unplanned outage may be computed separately. This goal should be combined with the other ones. Restoration speed plays a crucial role in many reliability indexes where time is usually combined with an outage size measure (e.g. number of customers, amount of unsupplied power). Similarly, there are also frequency indexes which measure how often an outage happen. Both time and frequency based indexes can be registered for a time-frame (e.g. for a month) and a geographical area. Perhaps the best known indexes are SAIDI (system average interruption duration index) and SAIFI (system average interruption frequency index) which respectively reflect how long and how often an average customer experiences an outage. These reliability criteria are not standardized and vary from country to country. As far as the authors know, there is no standardized reliability index that takes into consideration priority customers.

- Power quality: this can be referred as voltage quality since harmonics and current quality are often not taken into account during restoration. Also, impact of restoration actions on zonal power quality indices should considered. Amplitude is usually expressed as reasonable deviation in percentage from nominal voltage. The limits during restoration are generally more relaxed compared to normal operation. In the 
worst case, the voltage amplitude may go to zero for a long time period, we talk then about an outage, power loss or sustained interruption. The main objective of the restoration process is to have the smallest zone with power loss, for the smallest time period and with the lower frequency (thus it is related to the goals stated above). The causes have already been discussed in the introduction. When the voltage goes under the nominal value, we refer to it as voltage sag. This can be caused for example by a load that is too high for the feeder. Short time deviations are related to transient caused by abrupt changes in the network such as switching operations. Distribution companies consider more and more reliability indexes when proceeding to the restoration.

- Operational costs: the operator should limit power losses (balancing power between feeders, limiting current and reactive power by volt/VAr regulation, minimizing manual operations), and avoiding pecuniary penalties. National and/or regional regulator bodies make some reliability criteria compulsory where fines are specified in case the requirements are not satisfied. This pecuniary punishment allows to make a link with reliability indexes.

\section{Constraints}

During the restoration stage, operating (topological, switch requirement and loading capabilities) and power quality constraints should be respected. Here we give a short description of the restrictions:

- Topological restriction: radial structure allows savings because circuit-breakers and network elements can be designed to work with smaller values [2]. The drawback is that with this tree configuration, much larger areas lose supply [2]. On the contrary radial structure makes the localization easier. In the literature, radiality is usually considered a constraint. Because of some fundamental differences between overhead and underground power lines, they are not handled in the same way.

- Switch requirement: the switches should be operated according to allowed sequences [8], [9].

- Loading capabilities: the absolute limits expressed by engineer limits in terms of power/voltage/current for the transformers, busbars and lines. During emergency, some overloading is allowed for a limited time. Besides, the load should be within supply capacity [10]. Special care should be given to cold load pickup, because with the lack of diversity, the load may be two to five times higher than normal load [8], [11].
- Power quality: usually expressed as voltage range according to company code or according to regulation.

\section{Knowledge based approaches to assist power restoration}

An ideal restoration technique should be reliable, safe and efficient [2]. As we have seen, it should respect all constraints and optimize the goals. The restoration problem is a multi-objective constrained NP-hard combinatorial problem. To illustrate the combinatorial problem, let's take a network with ten tie-switches, we have then 10! (over 3.6 millions) different possible sequences [8]. It is true that some sequences may be pruned out because they are not valid. However, distributions networks generally have a three-figure number of switches [12]. For this reason, going through all combinations while optimizing the goals under constraints is prohibitive.

In the past, when the distribution networks were simpler, operators tended to rely on scenarios prepared in advance for all possible faults. But the networks soon became more complicated. In 1996, Curcic et al. already pointed that no rigid mathematical methodology has been proposed because of the complexity of the combinatorial problem [2]. Since it is an NP-hard problem, any strict mathematical method would have to over-simplify the problem or take prohibitive time.

Today, main techniques that are not mutually exclusive are:

- Heuristic rules: they are not based on a rigorous physical and mathematical proof but rather based on the operator experience and beliefs. For this reason there are also referred as knowledge-based techniques or expert systems. Due to their nature, they are highly specific to the characteristics of the network and the philosophy of the company.

Since they usually do not require complex mathematics nor advanced stochastic methods, papers in the early 1990s already formalized them [13]. However, heuristics rules have to be adapted with the evolution of network. For example the availability of more measures and more precise data means that information that has to be guessed heuristically can now be directly used. Moreover, the characteristics of the network and the priorities of the operators may evolve.

We will give some interesting heuristic rule describing the operations. They can be used in any knowledge base technique, assuming radial network constraints: at least a normally openswitch should be closed, use the nearest supporting feeder that has enough spare 
capacity, prefer operating normally closed switches that are nearby the isolated area, a given switch should be manipulated at most once, etc [13], [14].

Other heuristics are more related to the goals, for examples: restore first priority loads, restore as much load as possible, and minimize switching operations [7].

- Metheuristic techniques: they use random search (exploration) combined with heuristic (exploitation). Thus they are a kind of stochastic optimization methods. Here heuristic refers to the algorithm designer judgment (and not based on a mathematical proof) for the solution candidate while for heuristic rules, it is based on operator working on the field. This category includes ant colony (ACO), genetic (GA), immune (IA), simulated annealing (SA) algorithms and tabu search (TS). The designer of the algorithm has to find good heuristics, set the initial parameters (that may be generated randomly) and fine-tune empirically the parameters (since there are no formal rule) without guarantee that they can adapt well to new situations. Conversely, they may be well suited to explore large candidate space when you have no clue or little clue about the solution.

- Learning methods: these methods gradually increase their knowledge as they receive more inputs. They can be used in a supervised or unsupervised manner to learn. In the first category, pairs of inputs and outputs are provided. In contrast with metheuristic techniques on which papers appear on a regular basis, learning methods for distribution network restoration are barely dealt in the literature. Artificial neuronal networks (ANN) has generated little interest, while case based reasoning (CBR) has been hardly studied. The advantage of learning methods is that they improve their performance over time. However, they start to work well only after receiving a large number of inputs (a large training set or memory of cases)

Moreover, fuzzy and paraconsistent logic, Petri nets and multi-agent systems have been used to support these techniques. Fuzzy logic has been used to express the approximate expression of the heuristics rules or goals while paraconsistent logic allows to handle contradictions with a new logic [15], [16], [17]. Petri nets help represent the problem [7]. Multi-agent systems allow to lower the required bandwidth between the different devices and to alleviate the burden on the central system [18].

\section{Comparison}

Due to the evolution of networks, their differences across the world, the different restoration goals, there is no comprehensive benchmark of the available methods. There are two papers that compare respectively three and two metaheuristic algorithms [19], [9]. In Table I we give a comparison of some selected papers. We have included a reference on network reconfiguration to illustrate power quality issues and the use of IA. However, as we stated above restoration and reconfiguration can be done sequentially.

So far, distributed generation has received little attention. As a consequence, related techniques such as islanding (creating on purpose independent networks) or allowing power flow in more than one direction should also be studied more. Nevertheless, $\mathrm{Li}$ et al. allow the distribution network to work with islands during restoration [23]. Cold load pickup (CLPU) refers to the increase of load (up to 5 times normal load) for up to several hours when power is restored. This phenomenon is especially important when electricity is massively used for heating or cooling through devices regulated by thermostats [11]. Mohanty et al. estimated the restoration time based on CLPU modeled as a declining exponential [9].

Table I. - Comparison of some network features, restoration and reconfiguration goals from selected papers

\begin{tabular}{|c|c|c|c|c|c|c|c|c|c|}
\hline \multirow[t]{2}{*}{ Ref. } & \multirow[t]{2}{*}{$\begin{array}{l}\text { Me- } \\
\text { thod }\end{array}$} & \multicolumn{2}{|c|}{$\begin{array}{l}\text { Network } \\
\text { features }\end{array}$} & \multicolumn{3}{|c|}{ Restoration goals } & \multicolumn{3}{|c|}{$\begin{array}{l}\text { Reconfigu- } \\
\text { ration goals }\end{array}$} \\
\hline & & DG & CLPU & PC & SR & $\mathrm{PO}$ & $\mathrm{L}$ & LB & VL \\
\hline [20] & GA & & & $\mathrm{X}$ & $\mathrm{X}$ & $\mathrm{X}$ & & & \\
\hline [12] & $\begin{array}{l}\text { ACO } \\
+ \text { IA }\end{array}$ & & & & & & $\mathrm{X}$ & $\mathrm{X}$ & $\mathrm{X}$ \\
\hline [21] & $\begin{array}{r}\text { GA } \\
+ \text { SA }\end{array}$ & & & $\mathrm{X}$ & $\mathrm{X}$ & $\mathrm{X}$ & & & \\
\hline [9] & $\begin{array}{l}\text { ACO } \\
+ \text { SA }\end{array}$ & & $\mathrm{X}$ & & $\mathrm{X}$ & & & & \\
\hline [19] & $\begin{array}{l}\text { TS, } \\
\text { GA, } \\
\text { SA }\end{array}$ & & & & $\mathrm{X}$ & $\mathrm{X}$ & & & \\
\hline [7] & $\mathrm{ACO}$ & & $\mathrm{X}$ & $\mathrm{X}$ & $\mathrm{X}$ & & & & \\
\hline [22] & ANN & & & & $\mathrm{X}$ & $\mathrm{X}$ & & & \\
\hline [16] & CBR & & & & $\mathrm{X}$ & & & & \\
\hline [23] & IA & $\mathrm{X}$ & & & & $\mathrm{X}$ & & & $\mathrm{X}$ \\
\hline
\end{tabular}

(DG: distributed generation ; CLPU: cold load pickup ; PC: priority customers ; SR: speed of restoration ; PO: minimize power outage ; L: power loss ; LB: load balancing; VL: voltage level)

As for priority customers, Kumar et al. seem to consider only two cases: all priority customers are served or all priority customers are not served [20]. More interestingly, Hongshun and Xiangfei use a four-level categorization scheme as discussed above [7]. Ingaki et al. do refer to priority customers in the goals but do not include them in the experimental results [21].

Minimizing the switching operations was a goal in [7], [21] and [22]. To simplify the table, we associate this goal with minimizing the restoration duration. [21] and [20] interestingly make a distinction between manual and remote-controlled switches. Hsu et al. considered a network with manual switches, but against a background of ongoing automation process at the utility, where minimizing the switching operations has the additional benefit of limiting manpower and increasing the life expectancy of the device and so giving additional benefit 
of reducing operational costs [22]. The algorithm computation should also be taken into account if it is significant compared to the time required for operating the switches.

Minimizing the power outage size is perhaps the most obvious goal. This goal can be formulated in several ways (such as number of customer or expected energy not supplied) depending on several factors such as the metering capabilities of the company and the targeted reliability indexes. In the table, we considered the use of priority customers a more precise goal than outage size. That is why we did not put an additional $\mathrm{X}$ mark in the latter column.

The reconfiguration process, which can be done after an outage is restored or during normal operations, is done for reducing operation costs (power loss, load balancing) and improving power quality (voltage level). Power loss can be estimated with the impedance of the network. Reactive power should also be reduced to avoid power loss. Load balancing is important to reduce ageing of the equipment and to reduce the current levels and thus power loss. Voltage level configuration is an important power quality goal and usually is expressed by a percentage deviation to nominal values. Ahuja et al. optimized power loss, load balancing on transformer and voltage deviation [12]. Power loss was computed as the sum of Joule effects on each branch.

Lambert-Torres et al. devote most of their paper to explain the use of paraconsistent logic with CBR, but implemented it with a very simple network consisting of only one normally open tie switch [16]. Actually, it is not a network, but rather the configuration of a substation. So it remains to see, if the technique is scalable to a more realistic network and how all goals and restrictions can be dealt.

\section{Conclusion}

In this paper, we have seen the different stages to eliminate an outage, the different goals and constraints to consider during restoration. Next we have discussed the different techniques used during restoration. AI based techniques for distribution network restoration are still in their infancy. That is why, much work remains to be done in modelling more complex network features, taking into account more fine-grained goals and consider more restrictive constraints. As we have chiefly observed a lack of use of learning methods, we propose to investigate further CBR technique for power distribution systems restoration in a future work.

\section{Acknowledgment}

This research has been partially funded by a research contract between Universitat de Girona (the University of Girona) and Indra Software Labs in the framework of the CENIT Project ENERGOS-Tecnologías para la gestión automatizada e inteligente de las redes de distribución energética del futuro (CEN2009-1048)- granted by the
Spanish CDTI (Centro para el Desarrollo Tecnológico Industrial) and led by the utility Gas Natural Fenosa.

\section{References}

[1] M.M. Adibi, "Power System Restoration: methodologies\&Implementation strategies", IEEE press, New York (2000)

[2] S. Curcic, C.S. Özveren, L. Crowe, P.K.L Lo, "Electric power distribution network restoration: a survey of papers and a review of the restoration problem", in Electric Power Systen Research, No 35, pp. 73-86, 1996

[3] Thi Thu Ha Pham, Yvon Bésanger and Nouredine Hadjsaid, "New challenges in power system restoration with large scale of dispersed generation insertion”, in IEEE Transactions on power systems, Vol. 24, Issue 1, pp. 398-406, 2009

[4] Pedram Jahangiri and Mahmud Foutihi-Firuzabad, "Reliability assessment of distributed system with distribution generation”, in Power and Energy Conference, pp. 1551-1556, 2008

[5] U.G. Knight, "Power systems in emergencies: from contingency planning to crisis management", Wiley, Chichester (2001)

[6] Yogendra Kumar, Biswarup Das and Jaydev Sharma, "Genetic algorithm for supply restoration in distribution system with priority customers", in Proceeding of International Conference of Probabilistic Methods Applied to Power Systems, PMAPS 2006, pp. 1-7, 2006

[7] Shu Hongshun and Sun Xiangfei, "A novel approach to distribution network reconfiguration considering the priority of customers", in Proceedings of Transmission and Distribution Conference and Exhibition: Asia and Pacific, 2005 IEEE/PES 2005, pp. 1-4, 2005

[8] [Chavali, 2002], Sudhakar Chavali, Anil Pahwa and Sanjoy Das, "A genetic approach for optimal distribution feeder restoration during cold load pickup”, in Proceedings of the 2002 congress on evolutionary computation, pp. 1816-1819, 2002

[9] Indira Mohanty, Jugal Kalita, Sanjoy Das, Anil Pahwa, Erik Buehler, "Ant algorithm for the optimal restoration of distribution feeders during cold load pickup", in Proceedings of the 2003 IEEE Swarm Intelligence Symposium, 2003. SIS '03, pp. 132-137, 2003

[10] Isamu Watanabe and Ikuo Kurihara, "A hybrid genetic algorithm for service restoration in power distribution systems", in Proceedings of the IEEE congress on evolutionary computation, 2006. CEC 2006, pp. 3250-3257, 2006

[11] V. Kumar, H.C.R Kumar, I. Gupta, H.O. Gupta, "DG Integrated Approach for Service Restoration Under Cold Load Pickup”, in IEEE Transactions on Power Delivery, Volume 25, No 1, 2010

[12] Ashish Ahuja, Sanjoy Das and Anil Pahwa, “An AIS-ACO hybrid approach for multi-objective distribution system restoration", in IEEE Transactions on power systems, Vol. 22, No 3, pp. 1101-1111, 2007

[13] Y.-Y. Hsu, H.-M. Huang, H.-C Kuo, S.K Peng, C.W. Chang, K.J Chang, H.S Yu, C.E Chow and R.T. Kuo, 
"Distribution system service restoration using a heuristic search approach”, IEEE Transactions on Power Delivery, pp. 734-740, 1992

[14] Isamu Watanabe, “An ACO algorithm for service restoration in power distribution systems", in Proceedings of the 2005 IEEE congress on evolutionary computation, Vol. 3, pp.: 2864-2871, 2005.

[15] Yuan-Yih Hsu and Han-Ching Kuo, “A heuristic based fuzzy reasoning approach for distribution system service restoration”, in IEEE Transactions on Power Delivery, pp. 948 953, 1994

[16] G. Lambert-Torres, H.G. Martins, L.E. Borges da Silva, M.P. Coutinho and J.C Neto, "New technology applied to a restoration of electrical power substation", in Proceedings of Transmission \& Distribution Conference \& Exposition: Asia and Pacific, 2009, pp. 1-4, 2009.

[17] J.I. Da Silva Filho and A. Rocco, "Power systems outage possibilities analysis by paraconsistent logic", in Power and energy society general meeting - Conversion and delivery of electrical energy in the 21th century, 2008

[18] Rabie Belkacemi and Ali Feliachi, "Multi-agent design for power distribution system reconfiguration based on the artificial immune system algorithm”, in Proceedings of the 2010 symposium on circuits and systems, pp. 3461-3464, 2010
[19] Sakae Toune, Hiroyuki Fudo, Yoshikazu Fukuyama and Yosuke Nakanishi, "Comparative study of modern heuristic algorithms to service restoration in distribution systems", in IEEE Transactions on Power Delivery, Vol. 17, No 1, pp. 173181, 2002

[20] Y. Kumar, B. Das and J. Sharma, "Multiobjective, Multiconstraint Service Restoration of Electric Power Distribution System With Priority Customers", in IEEE Transactions on Power Delivery, pp. 261-270, 2008

[21] J. Inagaki, J. Nakajima and M. Haseyama, “A multiobjective service restoration method for power distribution systems”, in Proceedings of IEEE International Symposium on Circuits and Systems, 2006. ISCAS 2006, 2006

[22] Y.-Y. Hsu, and H.-M Huang, "Distribution system service restoration using the artificial neural network approach and pattern recognition method", in IEE Proceedings on Generation, Transmission and Distribution, Vol. 142, No 3, 1995

[23] X.D. Li, Y.Q. Xu, .L. Zhang, "Distribution service restoration with DGs based on multi-agent immune algorithm", in Proceedings of International Conference on Power Electronics and Intelligent Transportation System (PEITS), pp. 1-4, 2009. 